\title{
Removable Singularities for Anisotropic Elliptic Equations
}

\author{
Igor I. Skrypnik
}

Received: 4 November 2013 / Accepted: 30 April 2014 / Published online: 28 May 2014

(C) Springer Science+Business Media Dordrecht 2014

\begin{abstract}
We study a class of quasi-linear elliptic equations with model representative $\sum_{i=1}^{n}\left(\left|u_{x_{i}}\right|^{p_{i}-2} u_{x_{i}}\right)_{x_{i}}=0$, which solutions have singularities on a smooth manifold. We establish the condition for removability of singularity on a manifold for solutions of such equations.
\end{abstract}

Mathematics Subject Classifications (2010) 35B45 · 35J60

\section{Introduction and Main Result}

In this paper we study solutions to quasi-linear equations in the divergence form

$$
-\operatorname{div} \mathbf{A}(x, \nabla u)=a_{0}(x, \nabla u), \quad x \in \Omega \backslash \Gamma,
$$

where $\Omega$ is a domain in $\mathbb{R}^{n}, n \geq 3$ and $\Gamma \subset \Omega$ is a manifold of dimension $1 \leq s \leq n-2$.

Throughout the paper we suppose that the functions $\mathbf{A}: \Omega \times \mathbb{R}^{n} \rightarrow \mathbb{R}^{n}$ and $a_{0}: \Omega \times$ $\mathbb{R}^{n} \rightarrow \mathbb{R}^{n}$ are such that $\mathbf{A}(\cdot, \xi), a_{0}(\cdot, \xi)$ are Lebesgue measurable for all $\xi \in \mathbb{R}^{n}$, and $\mathbf{A}(x, \cdot), a_{0}(x, \cdot)$ are continuous for almost all $x \in \Omega, \mathbf{A}=\left(a_{1}, a_{2}, \ldots, a_{n}\right)$.

We also assume that the following structure conditions are satisfied:

$$
\begin{gathered}
\mathbf{A}(x, \xi) \xi \geq v_{1} \sum_{i=1}^{n}\left|\xi_{i}\right|^{p_{i}}, \\
\left|a_{i}(x, \xi)\right| \leq v_{2}\left(\sum_{j=1}^{n}\left|\xi_{j}\right|^{p_{j}}\right)^{1-\frac{1}{p_{i}}}, \quad i=\overline{1, n} \\
\left|a_{0}(x, \xi)\right| \leq v_{2}\left(\left(\sum_{i=1}^{n}\left|\xi_{i}\right|^{p_{i}}\right)^{1-\frac{1}{\alpha}}+1\right),
\end{gathered}
$$

I. I. Skrypnik ( $\square)$

Institute of Applied Mathematics and Mechanics of NAS of Ukraine,

R. Luxemburg tr. 74, 83114 Donetsk, Ukraine

e-mail: iskrypnik@iamm.donbass.com 
where $v_{1}, v_{2}$ are positive constants and

$$
\begin{gathered}
1<p_{1} \leq \cdots \leq p_{n-s}, \quad \frac{1}{\alpha}=\frac{1}{n-s} \sum_{i=1}^{n-s} \frac{1}{p_{i}}, \\
\alpha \leq p_{n-s+1} \leq \cdots \leq p_{n}, \quad \frac{1}{\beta}=\frac{1}{s} \sum_{i=n-s+1}^{n} \frac{1}{p_{i}}, \quad \frac{1}{p}=\frac{1}{n} \sum_{i=1}^{n} \frac{1}{p_{i}}, \\
\tilde{p}=\max \left(p_{n-s}, p_{n}\right)<\min \left(\frac{(n-1) p}{n-p}, \frac{n-s-1}{n-s-\alpha} \alpha\right), \quad \alpha<n-s .
\end{gathered}
$$

It is well known that the necessary and sufficient conditions for the harmonic function $u$ to have a removable singularity at $x_{0}$ is $u(x)=o\left(\left|x-x_{0}\right|^{2-n}\right)$ as $x \rightarrow x_{0}$. Until recently such a precise result for quasi-linear equations was known only for positive solutions since the celebrated paper by Serrin [14], under relevant assumptions on the coefficients in terms of $L^{q}$-spaces (see [18] for the survey of the relevant results). For the sign changing solutions Serrin's result is expressed in terms of $L^{q}$-conditions on the coefficients, and for removability of isolated singularities and singularities on the manifolds it leads to a more restrictive condition. A model example of the isotropic Eq. (1.1) is the following equation involving $p$-Laplacian

$$
-\Delta_{p} u=g u|u|^{p-2}+f \quad \text { in } \Omega \backslash \Gamma, p>1 .
$$

For $g, f \in L^{q}(\Omega), q>\frac{n}{p}$ Serrin's condition [13,14] on removability of singularities on manifold $\Gamma$ with dimension $s$ reduces to

$$
u(x)=O\left((d(x, \Gamma))^{-\frac{n-p-s}{p-1}+\delta}\right), \quad \delta>0, p<n-s,
$$

where $d(x, \Gamma)$ is the distance from point $x$ to the manifold $\Gamma$. Further analysis of sufficient conditions for removability of singularities of solutions has been made by many authors for different classes of nonlinear elliptic and parabolic equations (c.f., e.g [18] and references therein). The precise condition for the removability of singularity on the manifold $\Gamma$ for Eq. (1.6) with $g, f \in L^{q}(\Omega), q>\frac{n}{p}$ (and more general quasi-linear equations) has the form

$$
u(x)=o\left((d(x, \Gamma))^{-\frac{n-p-s}{p-1}}\right), \quad 1<p<n-s,
$$

which has been proved in [15]. In the case of an isolated singularity $(s=0)$ an analogous result was obtained in [12].

Equations of the form

$$
-\sum_{i=1}^{n}\left(\left|u_{x_{i}}\right|^{p_{i}-2} u_{x_{i}}\right)_{x_{i}}=g u|u|^{p-2}+f
$$

have not been much studied.

Examples constructed by Giaquinta [4] and Marcellini [9] show that Eq.(1.9) may have unbounded solutions if $p_{i} s$ are too far apart. Local boundedness of solutions to Eq. (1.9) has been obtained in $[3,6]$ under the condition

$$
1<p_{1} \leq \cdots \leq p_{n} \leq \frac{n p}{n-p}, \quad p<n
$$

This condition is sharp as there are unbounded solutions to Eq. (1.9) if condition Eq.(1.10) is violated (cf. [3, 6]). Local boundedness of the gradient of a solution to Eq. (1.9) was obtained in $[8,10]$ under condition Eq. (1.10) and sufficient smoothness of the coefficients. 
It is worth nothing that the explicit fundamental solution to Eq. (1.9) is unknown. Therefore until recently it has not been clear how a precise condition for the removability of an isolated singularity of a solution to Eq. (1.9) can be stated. This question was successfully answered in [11], where it was proved that a singularity at the point $\left\{x_{0}\right\}$ is removable if $g, f \in L^{q}(\Omega), q>\frac{n}{p}$, and

$$
\underset{D(R) \backslash D(r)}{\operatorname{ess} \sup _{(r)}}|u(x)|=o\left(r^{-\frac{n-p}{p-1}}\right), \quad p<n,
$$

where $R$ is some fixed number and

$$
\begin{gathered}
D(r)=\left\{x \in \Omega: \sum_{i=1}^{n}\left|x_{i}-x_{i}^{(0)}\right|^{a_{i}} \leq r\right\}, \\
a_{i}=\frac{p_{i}(p-1)}{p(n-1)-p_{i}(n-p)}, \quad i=1, \ldots, n, \\
1<p_{1} \leq \cdots \leq p_{n}<\frac{n-1}{n-p} p .
\end{gathered}
$$

Existence of the positive fundamental solution to equation Eq. (1.9) was proved in [2] under condition Eq. (1.14).

We are interested here in pointwise conditions on solutions to guarantee that the singularity on $\Gamma$ is removable, that is, the solution can be extended to $\Omega$. Before formulating the main results, let us remind the reader the definition of a weak solution to Eq. (1.1). Let $\Gamma$ be a manifold of class $C^{1}$ without boundary of dimension $s$ contained in $\Omega$. Without loss of generality assume that $\Gamma \subset\left\{x_{1}=x_{2}=\cdots=x_{n-s}=0\right\}$. We say that $u$ is a weak solution to Eq. (1.1) in $\Omega \backslash \Gamma$ if for an arbitrary function $\psi \in C^{1}(\Omega)$, vanishing in a neighborhood of $\Gamma$, we have the inclusion $u \psi \in W^{1, p_{1}, \ldots, p_{n}}(\Omega)$ and the integral identity

$$
\int_{\Omega}\left\{\mathbf{A}(x, \nabla u) \nabla(\varphi \psi)-a_{0}(x, \nabla u) \varphi \psi\right\} d x=0
$$

holds for any $\varphi \in \stackrel{\circ}{W^{1}}, p_{1}, \ldots, p_{n}(\Omega)$.

We say that a solution $u(x)$ of Eq. (1.1) has a removable singularity on the manifold $\Gamma$ if $u(x)$ can be extended to $\Gamma$ so that the extension $\tilde{u}(x)$ of $u(x)$ satisfies Eq. (1.1) in $\Omega$ and $u(x) \in W^{1, p_{1}, \ldots, p_{n}}(\Omega)$.

Let

$$
\begin{aligned}
& b_{i}:=\frac{p_{i}(\alpha-1)}{\alpha(n-s-1)-p_{i}(n-s-\alpha)}, \quad i=1, \ldots, n, \\
& x^{\prime}=\left(x_{1}, \ldots, x_{n-s}\right), \quad x^{\prime \prime}=\left(x_{n-s+1}, \ldots, x_{n}\right), \\
& \rho\left(x^{\prime}\right):=\left(\sum_{i=1}^{n-s}\left|x_{i}\right|^{\frac{b_{i}}{b_{1}}}\right)^{b_{1}}, \quad \rho\left(x^{\prime \prime}\right):=\left(\sum_{i=n-s+1}^{n}\left|x_{i}\right|^{\frac{b_{i}}{b_{1}}}\right)^{b_{1}} .
\end{aligned}
$$

For $R_{0}, H_{0}>0$ set

$$
\begin{gathered}
D\left(R_{0}, H_{0}\right)=\left\{x: \rho\left(x^{\prime}\right)<R_{0}, \rho\left(x^{\prime \prime}\right)<H_{0}\right\}, \\
D_{1}\left(R_{0}\right)=\left\{x^{\prime}: \rho\left(x^{\prime}\right)<R_{0}\right\}, \quad D_{2}\left(H_{0}\right)=\left\{x^{\prime \prime}: \rho\left(x^{\prime \prime}\right)<H_{0}\right\} .
\end{gathered}
$$

We can assume that $R_{0}, H_{0}$ are sufficiently small such that

$$
D\left(R_{0}, H_{0}\right) \subset \Omega, \quad \Gamma \subset D\left(R_{0}, \frac{H_{0}}{2}\right) \cap\left\{x^{\prime}=0\right\} .
$$


Next we define the number $M(r)$ characterizing local behaviour of the solution $u$ in the neighborhood of the manifold $\Gamma$.

$$
M(r):=\operatorname{ess} \sup \left\{|u(x)|: x \in D\left(R_{0}, H_{0}\right) \backslash D\left(r, H_{0}\right)\right\} .
$$

The regularity result from $[3,6]$ yields that $M(r)<\infty$ for $r>0$. Now we are ready to formulate our main result.

Theorem 1.1 Let $u$ be a weak solution to Eq. (1.1) in $\Omega \backslash \Gamma$. Let conditions Eq. (1.2)-(1.5) be fulfilled. Assume also that

$$
\lim _{r \rightarrow 0} r^{\frac{n-s-\alpha}{\alpha-1}} M(r)=0, \quad 1 \leq s \leq n-2 .
$$

Then a singularity of $u(x)$ on $\Gamma$ is removable.

Remark 1.1 In the critical case $\alpha=n-s$ the condition of removability on the manifold $\Gamma$ takes the form

$$
\lim _{r \rightarrow 0} m(r)|\ln r|^{-1}=0 \quad \text { (cf. [11]), }
$$

where $m(r)=\operatorname{ess} \sup \left\{|u(x)|: x \in \tilde{D}\left(R_{0}, H_{0}\right) \backslash \tilde{D}\left(r, H_{0}\right)\right\}, \tilde{D}\left(R_{0}, H_{0}\right)=\left\{x: d\left(x^{\prime}\right)<\right.$ $\left.R_{0}, d\left(x^{\prime \prime}\right)<H_{0}\right\}, d\left(x^{\prime}\right)=\left(\sum_{i=1}^{n-s}\left|x_{i}\right|^{\frac{p_{i}}{p_{1}}}\right)^{\frac{p_{1}}{n}}, d\left(x^{\prime \prime}\right)=\left(\sum_{i=n-s+1}^{n}\left|x_{i}\right|^{\frac{p_{i}}{p_{1}}}\right)^{\frac{p_{1}}{n}}$.

The result analogous to Theorem 1.1 can be proved for this case with respective changes in Lemmas 2.1-2.4 (see Section 2). We will not pursue this issue here.

The main step in proving Theorem 1.1 is the following result.

Theorem 1.2 Let the conditions of Theorem 1.1 be fulfilled. Then there exist positive constants $K_{0}, c$ depending only on $v_{1}, v_{2}, s, n, p_{1}, \ldots, p_{n}, R_{0}, H_{0}$ such that

$$
M(r) \leq K_{0} r^{-\frac{n-s-\alpha}{\alpha-1}+c}, \quad r>0 .
$$

We Point out that our approach continues the studies of I. V. Skrypnik [16, 17] on pointwise estimates of nonlinear capacity potentials. The rest of the paper contains the proof of the above theorems.

\section{Proof of Theorem 1.2}

\subsection{Auxiliary propositions}

The following lemmas will be used in the sequel. The first one is the well-known embedding lemma (see [1]).

Lemma 2.1 Let $\Omega \subset \mathbb{R}^{n}, n \geq 2$ be a bounded domain, $v \in \stackrel{\circ}{W}^{1,1}(\Omega)$ and

$$
\sum_{i=1}^{n} \int_{\Omega}|v|^{\alpha_{i}}\left|\frac{\partial v}{\partial x_{i}}\right|^{p_{i}} d x<\infty, \quad \alpha_{i} \geq 0, \quad p_{i} \geq 1 .
$$


If $1<p<n$, then $v \in L^{q}(\Omega), q=\frac{n p}{n-p}\left(1+\frac{1}{n} \sum_{i=1}^{n} \frac{\alpha_{i}}{p_{i}}\right)$ and the following inequality holds

$$
\|v\|_{L^{q}(\Omega)} \leq K_{1} \prod_{i=1}^{n}\left(\int_{\Omega}|v|^{\alpha_{i}}\left|\frac{\partial v}{\partial x_{i}}\right|^{p_{i}} d x\right)^{\frac{1}{n p_{i}\left(1+\frac{1}{n} \sum_{k=1}^{n} \frac{\alpha_{k}}{p_{k}}\right)}},
$$

where the constant $K_{1}$ depends only on $n, \alpha_{i}, p_{i}, i=1, \ldots, n$.

The next lemma is an immediate consequence of Lemma 2.1 .

Lemma 2.2 Let $v \in \stackrel{\circ}{W^{1,1}}(\Omega)$ and

$$
\sum_{i=1}^{n} \int_{\Omega}|v|^{-\alpha_{i}}\left|\frac{\partial v}{\partial x_{i}}\right|^{p_{i}} d x<\infty, \quad 0 \leq \alpha_{i}<p_{i}, \quad p_{i} \geq 1 .
$$

If $1<p<n$, then $v \in L^{q}(\Omega), q=\frac{n p}{n-p}\left(1-\frac{1}{n} \sum_{i=1}^{n} \frac{\alpha_{i}}{p_{i}}\right)$ and the following inequality holds

$$
\|v\|_{L^{q}(\Omega)} \leq K_{2} \prod_{i=1}^{n}\left(\int_{\Omega}|v|^{-\alpha_{i}}\left|\frac{\partial v}{\partial x_{i}}\right|^{p_{i}} d x\right)^{\frac{1}{n p_{i}\left(1-\frac{1}{n} \sum_{k=1}^{n} \frac{\alpha_{k}}{p_{k}}\right)}},
$$

with positive constant $K_{2}$ depending only on $n, \alpha_{i}, p_{i}, i=1, \ldots, n$.

In what follows we will frequently use the following lemma [7, Chapter II, Lemma 4.7].

Lemma 2.3 Let $\left\{y_{j}\right\}$ be a sequence of non-negative numbers such that for any $j=$ $0,1,2, \ldots$ the inequality

$$
y_{j+1} \leq C b^{j} y_{j}^{1+\varepsilon}
$$

holds with positive constants $\varepsilon, C>0, b>1$. Then the following estimate is true

$$
y_{j} \leq C^{\frac{(1+\varepsilon)^{j}-1}{\varepsilon}} b^{\frac{(1+\varepsilon)^{j}-1}{\varepsilon^{2}}-\frac{j}{\varepsilon}} y_{0}^{(1+\varepsilon)^{j}} .
$$

Particularly, if $y_{0} \leq C^{-\frac{1}{\varepsilon}} b^{-\frac{1}{\varepsilon^{2}}}$, then $\lim _{j \rightarrow \infty} y_{j}=0$.

2.2 Integral estimates for the gradient of solutions

Let $\tau \in C^{\infty}\left(\mathbb{R}^{1}\right)$ be such that $\tau(t)=0$ for $t \leq 1, \tau(t)=1$ for $t \geq 2,0 \leq \tau(t) \leq 1$, $0 \leq \frac{d \tau(t)}{d t} \leq 2, t \in \mathbb{R}^{1}$.

Fix a point $\left|\xi^{\prime \prime}\right| \leq \frac{H_{0}}{2}$, for $r>0, h>0$ set

$$
\psi_{r}\left(x^{\prime}\right)=\tau\left(r^{-1} \rho\left(x^{\prime}\right)\right), \quad \zeta_{h}\left(x^{\prime \prime}\right)=1-\tau\left(h^{-1} \rho\left(x^{\prime \prime}-\xi^{\prime \prime}\right)\right) .
$$

For $0<r \leq R_{0}$ set

$$
u_{r}=(u-M(r))_{+}, \quad E(r)=\left\{x \in D\left(R_{0}, H_{0}\right): u(x)>M(r)\right\} .
$$

By the known parameters we understand the numbers $v_{1}, v_{2}, n, s, p_{1}, \ldots, p_{n}, R_{0}$, $H_{0}, m$, where $m$ is a fixed positive number such that $m \geq 1+\tilde{p}$. In what follows $\gamma$ stands for a generic constant that depends on known parameters only and may vary from line to line. 
Lemma 2.4 Let the conditions of Theorem 1.2 be fulfilled. Then there exists a positive constant $c_{1}$ depending on the known parameters only such that the inequalities

$$
0<r<\rho \leq R_{0}, \quad 0<h \leq \frac{H_{0}}{2}, \quad \rho \leq h
$$

imply that

$$
\sum_{i=1}^{n} \int_{E(\rho)}\left|u_{x_{i}}\right|^{p_{i}} \psi_{r}^{m} \zeta_{h}^{m} d x \leq c_{1} M(r) h^{a_{s}}(\mu(r)+1)
$$

where $a_{s}=s\left(1+\frac{n-s-1}{\alpha-1}\left(\frac{\alpha}{\beta}-1\right)\right), \mu(r)=\sum_{i=1}^{n-s}\left(r^{\frac{n-s-\alpha}{\alpha-1}} M(r)\right)^{p_{i}-1}$.

Proof Without loss assume that $\lim _{r \rightarrow 0} M(r)=\infty$ and suppose that $R_{0}$ satisfies the additional condition $M\left(R_{0}\right) \geq 1$. Testing Eq. (1.15) by $\varphi=u_{\rho} \psi_{r}^{m-1} \zeta_{h}^{m}, \quad \psi=\psi_{r}$ and using conditions Eq. (1.2) we have

$$
\begin{aligned}
\sum_{i=1}^{n} \int_{E(\rho)}\left|u_{x_{i}}\right|^{p_{i}} \psi_{r}^{m} \zeta_{h}^{m} d x \leq & \gamma \sum_{i=1}^{n-s} \int_{E(\rho)}\left(\sum_{j=1}^{n}\left|u_{x_{j}}\right|^{p_{j}}\right)^{1-\frac{1}{p_{i}}} u_{\rho}\left|\frac{\partial \psi_{r}}{\partial x_{i}}\right| \psi_{r}^{m-1} \zeta_{h}^{m} d x \\
& +\gamma \sum_{i=n-s+1}^{n} \int_{E(\rho)}\left(\sum_{j=1}^{n}\left|u_{x_{j}}\right|^{p_{j}}\right)^{1-\frac{1}{p_{i}}} u_{\rho}\left|\frac{\partial \zeta_{h}}{\partial x_{i}}\right| \psi_{r}^{m} \zeta_{h}^{m-1} d x \\
& +\gamma \int\left(\left(\sum_{E(\rho)}^{n}\left|u_{x_{j}}\right|^{p_{j}}\right)^{1-\frac{1}{\alpha}}+1\right) u_{\rho} \psi_{r}^{m} \zeta_{h}^{m} d x .
\end{aligned}
$$

From this using Young's inequality we get

$$
\begin{aligned}
\sum_{i=1}^{n} \int_{E(\rho)}\left|u_{x_{i}}\right|^{p_{i}} \psi_{r}^{m} \zeta_{h}^{m} d x \leq & \gamma \sum_{i=1}^{n-s} \int_{E(\rho) \cap K(r)} u_{\rho}^{p_{i}}\left|\frac{\partial \psi_{r}}{\partial x_{i}}\right|^{p_{i}} \zeta_{h}^{m} d x \\
& +\gamma \sum_{i=n-s+1}^{n} \int_{E(\rho)} u_{\rho}^{p_{i}}\left|\frac{\partial \zeta_{h}}{\partial x_{i}}\right|^{p_{i}} \psi_{r}^{m} d x \\
& +\gamma \int_{E(\rho)} u_{\rho}^{\alpha} \psi_{r}^{m} \zeta_{h}^{m} d x+\gamma \int_{E(\rho)} \psi_{r}^{m} \zeta_{h}^{m} d x,
\end{aligned}
$$

where $K(r)=\left\{x^{\prime}: r<\rho\left(x^{\prime}\right)<2 r\right\}$.

Using the definition of $M(r)$ we have

$$
\begin{aligned}
\sum_{i=1}^{n-s} \int_{E(\rho) \cap K(r)} u_{\rho}^{p_{i}}\left|\frac{\partial \psi_{r}}{\partial x_{i}}\right|^{p_{i}} \zeta_{h}^{m} d x \leq & \gamma r^{n-s-\alpha} h^{a_{s}} \sum_{i=1}^{n-s} M^{p_{i}}(r) r^{-\frac{n-s-\alpha}{\alpha-1}\left(\alpha-p_{i}\right)} \\
& =\gamma M(r) h^{a_{s}} \sum_{i=1}^{n-s}\left(r^{\frac{n-s-\alpha}{\alpha-1}} M(r)\right)^{p_{i}-1} .
\end{aligned}
$$


Using condition Eq. (1.17) and inclusion $E(\rho) \subset D\left(\rho, H_{0}\right)$ we deduce

$$
\begin{aligned}
\sum_{i=n-s+1}^{n} \int_{E(\rho)} u_{\rho}^{p_{i}}\left|\frac{\partial \zeta_{h}}{\partial x_{i}}\right|^{p_{i}} \psi_{r}^{m} d x \leq & \gamma M(r) h^{a_{s}-\alpha} \sum_{i=n-s+1}^{n} h^{-\frac{n-s-\alpha}{\alpha-1}\left(\alpha-p_{i}\right)} \\
& \times \int_{r \leq \rho\left(x^{\prime}\right) \leq \rho} \rho^{-\frac{n-s-\alpha}{\alpha-1}\left(p_{i}-1\right)}\left(x^{\prime}\right) d x^{\prime} .
\end{aligned}
$$

Let introduce new independent variables

$$
x_{i}:=y_{i}^{\frac{\delta}{b_{i}}} \operatorname{sign} y_{i}, \quad y_{i}=1, \ldots, n, \delta=2 \max _{1 \leq i \leq n-s}\left(1, b_{i}\right),
$$

then after simple computation we get

$$
\begin{aligned}
& \int_{r \leq \rho\left(x^{\prime}\right) \leq \rho} \rho^{-\frac{n-s-\alpha}{\alpha-1}\left(p_{i}-1\right)}\left(x^{\prime}\right) d x^{\prime} \leq \gamma \int_{r \leq\left|y^{\prime}\right|^{\delta} \leq \rho}\left(\sum_{i=1}^{n-s}\left|y_{i}\right|^{\delta}\right)^{-\frac{n-s-\alpha}{\alpha-1}\left(p_{i}-1\right)} \prod_{i=1}^{n-s}\left|y_{i}\right|^{\frac{\delta}{b_{i}}-1} d y^{\prime} \\
& \leq \gamma \int_{0}^{\rho^{\frac{1}{\delta}}}\left|y^{\prime}\right|^{-\delta \frac{(n-s-\alpha)\left(p_{i}-1\right)}{\alpha-1}+\delta(n-s)-1} d\left|y^{\prime}\right| \\
& \leq \gamma \rho^{n-s-\frac{n-s-\alpha}{\alpha-1}\left(p_{i}-1\right)} \text {. }
\end{aligned}
$$

Therefore, using Eq. (1.5) we get

$$
\sum_{i=n-s+1}^{n} \int_{E(\rho)} u_{\rho}^{p_{i}}\left|\frac{\partial \zeta_{h}}{\partial x_{i}}\right|^{p_{i}} \psi_{r}^{m} d x \leq \gamma M(r) h^{a_{s}} \sum_{i=n-s+1}^{n}\left(\frac{\rho}{h}\right)^{\alpha+\frac{n-s-\alpha}{\alpha-1}\left(\alpha-p_{i}\right)} \leq \gamma M(r) h^{a_{s}} .
$$

Similarly

$$
\int_{E(\rho)} u_{\rho}^{\alpha} \psi_{r}^{m} \zeta_{h}^{m} d x \leq \gamma M(r) h^{a_{s}} \int_{r \leq \rho\left(x^{\prime}\right) \leq \rho} \rho^{-n+s+\alpha}\left(x^{\prime}\right) d x^{\prime} \leq \gamma M(r) h^{a_{s}} \rho^{\alpha} .
$$

The last term in the right-hand side of Eq. (2.8) we estimate using the inclusion $E(\rho) \subset$ $D\left(\rho, H_{0}\right)$. Thus collecting Eq. (2.8)-(2.12) we arrive at the required Eq. (2.7).

$$
\text { For } 0<\theta \rho<\rho \leq R_{0} \text { set }
$$

$$
E(\theta \rho, \rho)=\{x \in E(\rho): u(x)<M(\theta \rho)\}, \quad u^{(\theta \rho)}(x)=\min \left\{u_{\rho}(x), M(\theta \rho)-M(\rho)\right\} .
$$

Lemma 2.5 Let the conditions of Theorem 1.2 be fulfilled. Then there exists a positive constant $c_{2}$ depending on the known parameters only such that the inequalities

$$
\theta \in(0,1), 0<\lambda<\min \left(1, \frac{\alpha(\alpha-1)}{n-s-\alpha}\right), 0<r<\frac{\theta \rho}{2}<\rho \leq R_{0}, \rho \leq h
$$


imply that

$$
\begin{aligned}
\sum_{i=1}^{n} \int_{E(\theta \rho, \rho)} u_{\rho}^{\lambda-1}\left|u_{x_{i}}\right|^{p_{i}} \psi_{r}^{m} \zeta_{h}^{m} d x \leq & c_{2}(M(\theta \rho)-M(\rho))^{\frac{\lambda p_{n}}{p_{n}-1}} \sum_{i=1}^{n} \\
& \times \int_{E(\theta \rho)} u_{\rho}^{-1-\frac{\lambda}{p_{n}-1}}\left|u_{x_{i}}\right|^{p_{i}} \psi_{r}^{m} \zeta_{h}^{m} d x \\
& +c_{2} \sum_{i=n-s+1}^{n} \int_{E(\rho)} u_{\rho}^{p_{i}-1+\lambda \mid}\left|\frac{\partial \zeta_{h}}{\partial x_{i}}\right|^{p_{i}} \psi_{r}^{m} d x \\
& +c_{2} \int_{E(\rho)} u_{\rho}^{\alpha-1+\lambda} \psi_{r}^{m} \zeta_{h}^{m} d x \\
& +c_{2}(\theta \rho)^{-\frac{\lambda(n-s-\alpha)}{\alpha-1}} \rho^{n-s} h^{a_{s}}+c_{2} \mu_{1}(r)(\theta \rho)^{-\frac{\lambda(n-s-\alpha)}{\alpha-1}} h^{a_{s}},
\end{aligned}
$$

where $\mu_{1}(r)=\sum_{i=1}^{n-s}\left(r^{\frac{n-s-\alpha}{\alpha-1}} M(r)\right)^{1-\frac{1}{p_{i}}}$, and $a_{s}$ was defined in Lemma 2.4.

Proof Test Eq. (1.15) by $\varphi=\left(u^{(\theta \rho)}\right)^{\lambda} \psi_{r}^{m-1} \zeta_{h}^{m}, \quad \psi=\psi_{r}$. Using Eq. (1.2) we have

$$
\begin{aligned}
\sum_{i=1}^{n} \int_{E(\theta \rho, \rho)} u_{\rho}^{\lambda-1}\left|u_{x_{i}}\right|^{p_{i}} \psi_{r}^{m} \zeta_{h}^{n} d x \leq & \gamma \sum_{i=1}^{n-s} \int_{E(\rho) \cap K(r)}\left(\sum_{j=1}^{n}\left|u_{x_{j}}\right|^{p_{j}}\right)^{1-\frac{1}{p_{i}}}\left(u^{(\theta \rho)}\right)^{\lambda}\left|\frac{\partial \psi_{r}}{\partial x_{i}}\right| \psi_{r}^{m-1} \zeta_{h}^{m} d x \\
& +\gamma \sum_{i=n-s+1}^{n} \int_{E(\rho)}\left(\sum_{j=1}^{n}\left|u_{x_{j}}\right|^{p_{j}}\right)^{1-\frac{1}{p_{i}}}\left(u^{(\theta \rho)}\right)^{\lambda}\left|\frac{\partial \zeta_{h}}{\partial x_{i}}\right| \psi_{r}^{m} \zeta_{h}^{m-1} d x \\
& +\gamma \int_{E(\rho)}\left(\left(\sum_{j=1}^{n}\left|u_{x_{j}}\right|^{p_{j}}\right)^{1-\frac{1}{\alpha}}+1\right)\left(u^{(\theta \rho)}\right)^{\lambda} \psi_{r}^{m} \zeta_{h}^{m} d x=I_{1}+I_{2}+I_{3} .
\end{aligned}
$$

First we estimate $I_{1}$. By the Hölder inequality, Eq. (1.17) and Lemma 2.4 we obtain

$$
\begin{aligned}
I_{1} \leq & \gamma M^{\lambda}(\theta \rho) \sum_{i=1}^{n-s}\left(\sum_{j=1}^{n} \int_{E(\rho)}\left|u_{x_{i}}\right|^{p_{i}} \psi_{r}^{m} \zeta_{h}^{n} d x\right)^{1-\frac{1}{p_{i}}}\left(\int_{E(\rho)}\left|\frac{\partial \psi_{r}}{\partial x_{i}}\right|^{p_{i}} \zeta_{h}^{m} d x\right)^{\frac{1}{p_{i}}} \\
& \leq \gamma(\theta \rho)^{-\frac{\lambda(n-s-\alpha)}{\alpha-1}} h^{a_{s}} \sum_{i=1}^{n-s}\left(r^{\frac{n-s-\alpha}{\alpha-1}} M(r)(1+\mu(r))\right)^{1-\frac{1}{p_{i}}} \leq \gamma(\theta \rho)^{-\frac{\lambda(n-s-\alpha)}{\alpha-1}} h^{a_{s}} \mu_{1}(r) .
\end{aligned}
$$

To estimate $I_{2}$ we decompose $E(\rho)$ as $E(\rho)=E(\theta \rho, \rho) \cup E(\theta \rho)$. By the Young inequality and using the evident inequality $u_{\rho}^{-1} \leq(M(\theta \rho)-M(\rho))^{-1}$ for $x \in E(\theta \rho)$, we have 


$$
\begin{aligned}
I_{2}-\frac{1}{4} \sum_{i=1}^{n} \int_{E(\theta \rho, \rho)} u_{\rho}^{\lambda-1}\left|u_{x_{i}}\right|^{p_{i}} \psi_{r}^{m} \zeta_{h}^{m} d x \leq & \gamma \sum_{i=n-s+1}^{n} \int_{E(\theta \rho, \rho)} u_{\rho}^{p_{i}-1+\lambda \mid}\left|\frac{\partial \zeta_{h}}{\partial x_{i}}\right|^{p_{i}} \psi_{r}^{m} d x \\
& +\gamma \sum_{i=n-s+1}^{n}(M(\theta \rho)-M(\rho))^{\frac{\lambda p_{i}}{p_{i}-1}} \\
& \times \int_{E(\theta \rho)} u_{\rho}^{-1-\frac{\lambda}{p_{i}-1}}\left|u_{x_{i}}\right|^{p_{i}} \psi_{r}^{m} \zeta_{h}^{m} d x \\
+ & \sum_{i=n-s+1}^{n} \int_{E(\theta \rho)} u_{\rho}^{p_{i}-1+\lambda \mid}\left|\frac{\partial \zeta_{h}}{\partial x_{i}}\right|^{p_{i}} \psi_{r}^{n} d x \\
\leq & \gamma(M(\theta \rho)-M(\rho))^{\frac{\lambda p_{n}}{p_{n}-1}} \sum_{i=1}^{n} \\
& \times \int_{E(\theta \rho)} u_{\rho}^{-1-\frac{\lambda}{p_{n}-1}}\left|u_{x_{i}}\right|^{p_{i}} \psi_{r}^{m} \zeta_{h}^{m} d x \\
& +\gamma \sum_{i=n-s+1}^{n} \int_{E(\rho)} u_{\rho}^{p_{i}-1+\lambda \mid}\left|\frac{\partial \zeta_{h}}{\partial x_{i}}\right|^{p_{i}} \psi_{r}^{m} d x .
\end{aligned}
$$

Similarly to Eq. (2.16) we have

$$
\begin{aligned}
I_{3} \leq \frac{1}{4} \sum_{i=1}^{n} \int_{E(\theta \rho, \rho)} u_{\rho}^{\lambda-1}\left|u_{x_{i}}\right|^{p_{i}} \psi_{r}^{m} \zeta_{h}^{m} d x+ & \gamma(M(\theta \rho)-M(\rho))^{\frac{\lambda p_{n}}{p_{n}-1}} \sum_{i=1}^{n} \\
& \times \int_{E(\theta \rho)} u_{\rho}^{-1-\frac{\lambda}{p_{n}-1}}\left|u_{x_{i}}\right|^{p_{i}} \psi_{r}^{m} \zeta_{h}^{m} d x \\
& +\gamma \int_{E(\rho)} u_{\rho}^{\alpha-1+\lambda} \psi_{r}^{m} \zeta_{h}^{m} d x \\
& +\gamma(\theta \rho)^{-\frac{\lambda(n-s-\alpha)}{\alpha-1}} \rho^{n-s} h^{a_{s}} .
\end{aligned}
$$

Collecting Eq. (2.14)-(2.17) we arrive at the required Eq. (2.13).

Lemma 2.6 Let the conditions of Lemma 2.5 be fulfilled. Then there exists a positive number $c_{3}$ depending on the known parameters only such that

$$
\begin{aligned}
& \sum_{i=1}^{n} \int_{E(\theta \rho)} u_{\rho}^{-1-\frac{\lambda}{p_{n}-1}}\left|u_{x_{i}}\right|^{p_{i}} \psi_{r}^{m} \zeta_{h}^{m} d x \leq c_{3}(M(\theta \rho)-M(\rho))^{-\frac{\lambda p_{n}}{p_{n}-1}} \sum_{i=n-s+1}^{n} \\
& \times \int_{E(\rho)} u_{\rho}^{p_{i}-1+\lambda}\left|\frac{\partial \zeta_{h}}{\partial x_{i}}\right|^{p_{i}} \psi_{r}^{m} d x \\
&+c_{3}(M(\theta \rho)-M(\rho))^{-\frac{\lambda p_{n}}{p_{n}-1}} \int_{E(\rho)} u_{\rho}^{\alpha-1+\lambda} \psi_{r}^{m} \zeta_{h}^{m} d x \\
&+c_{3}(M(\theta \rho)-M(\rho))^{-\frac{\lambda}{p_{n}-1}} \mu_{1}(r)+c_{3}(M(\theta \rho) \\
&-M(\rho))^{-\frac{\lambda}{p_{n}-1}} \rho^{n-s} h^{a_{s}},
\end{aligned}
$$


where $\mu_{1}(s)$ was defined in Lemma 2.5.

Proof Test Eq. (1.15) by

$$
\varphi=\left[(M(\theta \rho)-M(\rho))^{-\frac{\lambda}{p_{n}-1}}-\max ^{-\frac{\lambda}{p_{n}-1}}\left(u_{\rho}, M(\theta \rho)-M(\rho)\right)\right] \psi_{r}^{m-1} \zeta_{h}^{m}, \quad \psi=\psi_{r},
$$

using Eq. (1.2) and the Young inequality we have

$$
\begin{aligned}
& \sum_{i=1}^{n} \int_{E(\theta \rho)} u_{\rho}^{-1-\frac{\lambda}{p_{n}-1}}\left|u_{x_{i}}\right|^{p_{i}} \psi_{r}^{m} \zeta_{h}^{m} d x \\
& \quad \leq \gamma(M(\theta \rho)-M(\rho))^{-\frac{\lambda}{p_{n}-1}} \sum_{i=1}^{n-s} \int_{E(\rho) \cap K(r)}\left(\sum_{j=1}^{n}\left|u_{x_{j}}\right|^{p_{j}}\right)^{1-\frac{1}{p_{i}}}\left|\frac{\partial \psi_{r}}{\partial x_{i}}\right| \psi_{r}^{m-1} \zeta_{h}^{m} d x \\
& \quad+\gamma \sum_{i=n-s+1}^{n}(M(\theta \rho)-M(\rho))^{-\frac{\lambda p_{i}}{p_{n}-1}} \int_{E(\theta \rho)} u_{\rho}^{\frac{\lambda\left(p_{i}-1\right)}{p_{n}-1}+p_{i}-1}\left|\frac{\partial \zeta_{h}}{\partial x_{i}}\right|^{p_{i}} \psi_{r}^{m} d x \\
& \quad+\gamma(M(\theta \rho)-M(\rho))^{-\frac{\lambda \alpha}{p_{n}-1}} \int_{E(\theta \rho)} u_{\rho}^{\frac{\lambda(\alpha-1)}{p_{n}-1}+\alpha-1} \psi_{r}^{m} \zeta_{h}^{m} d x+\gamma(M(\theta \rho)-M(\rho))^{-\frac{\lambda}{p_{n}-1}} \\
& \quad \times \int_{E(\theta \rho)} \psi_{r}^{m} \zeta_{h}^{m} d x .
\end{aligned}
$$

The first term in the right-hand side of Eq. (2.19) has been estimated in Eq. (2.15), therefore we arrive at the required Eq. (2.18).

Combining Lemmas 2.5, 2.6 we get

Theorem 2.1 Let the conditions of Theorem 1.2 be fulfilled. Then there exists a positive constant $c_{4}$ depending on the known parameters only such that the inequalities $0<\theta<$ $1,0<\lambda<\min \left(1, \frac{\alpha(\alpha-1)}{n-s-\alpha}\right), 0<r<\frac{\theta \rho}{2}<\rho \leq R_{0}, \rho \leq h$ imply that

$$
\begin{aligned}
& \sum_{i=1}^{n} \int_{E(\theta \rho, \rho)} u_{\rho}^{\lambda-1}\left|u_{x_{i}}\right|^{p_{i}} \psi_{r}^{m} \zeta_{h}^{m} d x \leq c_{4} \sum_{i=n-s+1}^{n} \int_{E(\rho)} u_{\rho}^{p_{i}-1+\lambda}\left|\frac{\partial \zeta_{h}}{\partial x_{i}}\right|^{p_{i}} \psi_{r}^{m} d x \\
& +c_{4} \int_{E(\rho)} u_{\rho}^{\alpha-1+\lambda} \psi_{r}^{m} \zeta_{h}^{m} d x+c_{4} G(r, \rho, h),
\end{aligned}
$$

where $G(r, \rho, h)=(\theta \rho)^{-\frac{\lambda(n-s-\alpha)}{\alpha-1}} \rho^{n-s} h^{a_{s}}+(\theta \rho)^{-\frac{\lambda(n-s-\alpha)}{\alpha-1}} h^{a_{s}} \mu_{1}(r)$.

2.3 Integral estimates of solutions

Let

$$
\frac{n-s}{n-s-\left(p_{n}-\alpha\right) \frac{n-s-\alpha}{\alpha-1}}<q<\frac{n-s}{n-s-\alpha}
$$


set

$$
I(\rho, h)=\rho^{(n-s) \frac{q-1}{q}} \int_{D_{2}\left(H_{0}\right)} d x^{\prime \prime}\left(\int_{D_{1}\left(R_{0}\right)}\left(u_{\rho}^{\alpha-1+\lambda} \psi_{r}^{m} \zeta_{h}^{m}\right)^{q} d x^{\prime}\right)^{\frac{1}{q}}
$$

Lemma 2.7 Let the conditions of Theorem 1.2 be fulfilled and $0<\lambda<$ $\min \left(1, \frac{\alpha(\alpha-1)}{n-s-\alpha}, \frac{\alpha(n-s-1)}{n-s-\alpha}-p_{n}\right)$. Then there exists a positive constant $c_{5}$ depending on the known parameters only such that

$$
I(\rho, h) \leq 2^{\alpha-1+\lambda} \theta^{-(n-s) \frac{q-1}{q}} I(\theta \rho, h)+c_{5}\left(\frac{\rho}{h}\right)^{\alpha-\frac{n-s-\alpha}{\alpha-1}\left(p_{n}-\alpha\right)} I(\rho, 2 h)+c_{5} G_{1}(r, \rho, h),
$$

where

$$
\begin{aligned}
G_{1}(r, \rho, h)= & (\theta \rho)^{-\frac{\lambda(n-s-\alpha)}{\alpha-1}} \rho^{n-s+\alpha} h^{a_{s}} \\
& +(\theta \rho)^{-\frac{\lambda(n-s-\alpha)}{\alpha-1}} \rho^{\alpha} h^{a_{s}}\left(\sum_{i=n-s+1}^{n}\left(r^{\frac{n-s-\alpha}{\alpha-1}} M(r)\right)^{p_{i}-1}+\sum_{i=n-s+1}^{n}\left(r^{\frac{n-s-\alpha}{\alpha-1}} M(r)\right)^{1-\frac{1}{p_{i}}}\right) \\
& +\rho^{\alpha} h^{a_{s}-\frac{\alpha(n-s-1)}{\alpha-1}} \sum_{i=n-s+1}^{n} h^{\frac{h-s-\alpha}{\alpha-1} p_{i}}\left(r^{\frac{n-s-\alpha}{\alpha-1}} M(r)\right)^{p_{i}-1+\lambda} \\
& +\rho^{\alpha} h^{a_{s}}\left(r^{\frac{n-s-\alpha}{\alpha-1}} M(r)\right)^{\alpha-1+\lambda} .
\end{aligned}
$$

Proof Let $\chi(E(\theta \rho, \rho)), \chi(E(\theta \rho))$ denote the characteristic functions of the sets $E(\theta \rho, \rho)$, $E(\theta \rho)$ respectively. We will estimate $I(\rho, h)$ using the inequality

$$
\begin{aligned}
u_{\rho}^{\alpha-1+\lambda} \leq & u_{\rho}^{\alpha-1+\lambda} \chi(E(\theta \rho, \rho))+2^{\alpha-1+\lambda}\left(u_{\theta \rho}^{\alpha-1+\lambda}+(M(\theta \rho)-M(\rho))^{\alpha-1+\lambda}\right) \chi(E(\theta \rho)) \\
& \leq 2^{\alpha-1+\lambda} u_{\theta \rho}^{\alpha-1+\lambda} \chi(E(\theta \rho))+2^{\alpha-1+\lambda}\left(u^{(\theta \rho)}\right)^{\alpha-1+\lambda}, \quad x \in E(\rho) .
\end{aligned}
$$

Thus

$$
I(\rho, h) \leq 2^{\alpha-1+\lambda} \theta^{-(n-s) \frac{q-1}{q}} I(\theta \rho, h)+\gamma \rho^{(n-s) \frac{q-1}{q}} I_{4},
$$

where

$$
I_{4}=\int_{D_{2}\left(H_{0}\right)} d x^{\prime \prime}\left(\int_{D_{1}\left(R_{0}\right)}\left(\left(u^{(\theta \rho)}\right)^{\alpha-1+\lambda} \psi_{r}^{m} \zeta_{h}^{m}\right)^{q} d x^{\prime}\right)^{\frac{1}{q}} .
$$

Using the Hölder inequality and Lemma 2.2 with $\alpha_{1}=\cdots=\alpha_{n-s}=1-\lambda$, and choosing $m$ from the condition $m \frac{p_{1}-1+\lambda}{\alpha-1+\lambda}-\tilde{p} \geq 1$, we obtain

$$
\begin{aligned}
I_{4} \leq & \gamma \rho^{-(n-s) \frac{q-1}{q}+\alpha} \int_{D_{2}\left(H_{0}\right)} d x^{\prime \prime}\left(\int_{D_{1}\left(R_{0}\right)}\left(\left(u^{(\theta \rho)}\right)^{\alpha-1+\lambda} \psi_{r}^{m} \zeta_{h}^{m}\right)^{\frac{n-s}{n-s-\alpha}} d x^{\prime}\right)^{\frac{n-s-\alpha}{n-s}} \\
& \leq \gamma \rho^{-(n-s) \frac{q-1}{q}+\alpha} \sum_{i=1}^{n-s} \int_{E(\theta \rho, \rho)} u_{\rho}^{\lambda-1}\left|u_{x_{i}}\right|^{p_{i}} \psi_{r}^{m \frac{p_{1}-1+\lambda}{\alpha-1+\lambda}} \zeta_{h}^{m \frac{p_{1}-1+\lambda}{\alpha-1+\lambda}} d x \\
& +\gamma \rho^{-(n-s) \frac{q-1}{q}+\alpha} \sum_{i=1}^{n-s} \int_{E(\rho)}\left(u^{(\theta \rho)}\right)^{p_{i}-1+\lambda}\left|\frac{\partial \psi_{r}}{\partial x_{i}}\right|^{p_{i}} \zeta_{h}^{m \frac{p_{1}-1+\lambda}{\alpha-1+\lambda}} d x
\end{aligned}
$$


By Theorem 2.1

$$
\begin{aligned}
& \sum_{i=1}^{n-s} \int_{E(\theta \rho, \rho)} u_{\rho}^{\lambda-1}\left|u_{x_{i}}\right|^{p_{i}} \psi_{r}^{m \frac{p_{1}-1+\lambda}{\alpha-1+\lambda}} \zeta_{h}^{m \frac{p_{1}-1+\lambda}{\alpha-1+\lambda}} d x \\
& \quad \leq \gamma \sum_{i=n-s+1}^{n} \int_{E(\rho)} u_{\rho}^{p_{i}-1+\lambda}\left|\frac{\partial \zeta_{h}}{\partial x_{i}}\right|^{p_{i}} \psi_{r}^{m \frac{p_{1}-1+\lambda}{\alpha-1+\lambda}} d x+\gamma \int_{E(\rho)} u_{\rho}^{\alpha-1+\lambda} \psi_{r}^{m \frac{p_{1}-1+\lambda}{\alpha-1+\lambda}} \zeta_{h}^{m \frac{p_{1}-1+\lambda}{\alpha-1+\lambda}} d x \\
& \quad+\gamma G(r, \rho, h) .
\end{aligned}
$$

From this and form the fact that $\left\{\zeta_{h} \neq 0\right\} \subseteq\left\{\zeta_{2 h}=1\right\}$ we obtain

$$
\begin{aligned}
I_{4} \leq & \gamma \rho^{-(n-s) \frac{q-1}{q}+\alpha} \sum_{i=1}^{n-s} \int_{E(\rho)}\left(u^{(\theta \rho)}\right)^{p_{i}-1+\lambda}\left|\frac{\partial \psi_{r}}{\partial x_{i}}\right|^{p_{i}} \zeta_{h} d x+\gamma \rho^{-(n-s) \frac{q-1}{q}+\alpha} \sum_{i=n-s+1}^{n} \\
& \times \int_{E(\rho)} u_{\rho}^{p_{i}-1+\lambda}\left|\frac{\partial \zeta_{h}}{\partial x_{i}}\right|^{p_{i}} \psi_{r} \zeta_{2 h}^{m} d x \\
& +\gamma \rho^{-(n-s) \frac{q-1}{q}+\alpha} \int_{E(\rho)} u^{\alpha-1+\lambda} \psi_{r} \zeta_{2 h}^{m} d x+\gamma \rho^{-(n-s) \frac{q-1}{q}+\alpha} G(r, \rho, h) .
\end{aligned}
$$

Similarly to Eq. (2.9) we have

$$
\sum_{i=1}^{n-s} \int_{E(\rho)}\left(u^{(\theta \rho)}\right)^{p_{i}-1+\lambda}\left|\frac{\partial \psi_{r}}{\partial x_{i}}\right|^{p_{i}} \zeta_{h} d x \leq \gamma(\theta \rho)^{-\frac{\lambda(n-s-\alpha)}{\alpha-1}} h^{a_{s}} \sum_{i=1}^{n-s}\left(r^{\frac{n-s-\alpha}{\alpha-1}} M(r)\right)^{p_{i}-1} .
$$

Using Eq. (1.17) and the Hölder inequality we obtain

$$
\begin{gathered}
\sum_{i=n-s+1}^{n} \int_{E(\rho)} u^{p_{i}-1+\lambda}\left|\frac{\partial \zeta_{h}}{\partial x_{i}}\right|^{p_{i}} \psi_{r}^{m} \zeta_{2 h}^{m} d x \\
\leq \gamma h^{a_{s}-\alpha \frac{n-s-1}{\alpha-1}} \sum_{i=n-s+1}^{n} h^{\frac{n-s-\alpha}{\alpha-1} p_{i}}\left(r^{\frac{n-s-\alpha}{\alpha-1}} M(r)\right)^{p_{i}-1+\lambda} r^{\frac{n-s-1}{\alpha-1} \alpha-\frac{n-s-\alpha}{\alpha-1}\left(p_{n}+\lambda\right)} \\
+\gamma \sum_{i=n-s+1}^{n} \int_{E(\rho) \backslash K(r)} \rho^{-\frac{n-s-\alpha}{\alpha-1}\left(p_{i}-\alpha\right)}\left(x^{\prime}\right) u_{\rho}^{\alpha-1+\lambda}\left|\frac{\partial \zeta_{h}}{\partial x_{i}}\right|^{p_{i}} \psi_{r}^{m} \zeta_{2 h}^{m} d x \\
\leq \gamma h^{a_{s}-\alpha \frac{n-s-1}{\alpha-1}} \sum_{i=n-s+1}^{n} h^{\frac{n-s-\alpha}{\alpha-1} p_{i}}\left(r^{\frac{n-s-\alpha}{\alpha-1}} M(r)\right)^{p_{i}-1+\lambda} \\
\quad+\gamma I(\rho, 2 h) \sum_{i=n-s+1}^{n} h^{-\alpha-\frac{n-s-\alpha}{\alpha-1}\left(\alpha-p_{i}\right)} \rho^{-\frac{n-s-\alpha}{\alpha-1}\left(p_{i}-\alpha\right)} \\
\sum_{i=n-s+1}^{n} h^{\frac{n-s-\alpha}{\alpha-1} p_{i}}\left(r^{\frac{n-s-\alpha}{\alpha-1}} M(r)\right)^{p_{i}-1+\lambda}+\gamma \rho^{-\alpha}\left(\frac{\rho}{h}\right)^{\alpha-\frac{n-s-\alpha}{\alpha-1}\left(p_{n}-\alpha\right)} I(\rho, 2 h) .
\end{gathered}
$$


Similarly to Eq. (2.30) we have

$$
\int_{E(\rho)} u_{\rho}^{\alpha-1+\lambda} \psi_{r}^{m} \zeta_{2 h}^{m} d x \leq \gamma h^{a_{s}}\left(r^{\frac{n-s-\alpha}{\alpha-1}} M(r)\right)^{\alpha-1+\lambda}+\gamma \rho^{(n-s) \frac{q-1}{q}} I(\rho, 2 h) .
$$

Combining estimates Eq. (2.26)-(2.31) we arrive at the required Eq. (2.25).

We choose $\lambda$ such that

$$
0<\lambda<\min \left(1, \frac{\alpha(n-s-1)}{n-s-\alpha}-p_{n}, \frac{\alpha-1}{n-s-\alpha}\left(\alpha-(n-s) \frac{q-1}{q}\right)\right) .
$$

Theorem 2.2 Let the conditions of Theorem 1.2 be fulfilled. Then there exist positive numbers $c_{6}, c_{7}$ depending on the known parameters only such that the inequalities

$$
0<r<2 \rho \leq R_{0}, \quad \rho \leq c_{6} h
$$

imply that

$$
I(\rho, h) \leq c_{7} h^{n+a_{s}-s} \rho^{\alpha-\frac{\lambda(n-s-\alpha)}{\alpha-1}}+c_{7} h^{a_{s}} \rho^{n-s+\alpha-\frac{\lambda(n-s-\alpha)}{\alpha-1}}+c_{7} G_{2}(r, \rho, h),
$$

where

$$
\begin{aligned}
& G_{2}(r, \rho, h)=\rho^{\alpha-\frac{\lambda(n-s-\alpha)}{\alpha-1}} h^{a_{s}}\left\{\sum_{i=1}^{n-s}\left(r^{\frac{n-s-\alpha}{\alpha-1}} M(r)\right)^{p_{i}-1}+\sum_{i=1}^{n-s}\left(r^{\frac{n-s-\alpha}{\alpha-1}} M(r)\right)^{1-\frac{1}{p_{i}}}\right. \\
& \left.+\sum_{i=n-s 1}^{n} h^{-\alpha \frac{n-s-1}{\alpha-1}+\frac{n-s-\alpha}{\alpha-1} p_{i}}\left(r^{\frac{n-s-\alpha}{\alpha-1}} M(r)\right)^{p_{i}-1+\lambda}+\left(r^{\frac{n-s-\alpha}{\alpha-1}} M(r)\right)^{\alpha-1+\lambda}\right\} .
\end{aligned}
$$

Proof Let $A=2^{\alpha-1+\lambda} \theta^{-(n-s) \frac{q-1}{q}}, B=c_{5} c_{6}^{\alpha-\frac{n-s-\alpha}{\alpha-1}\left(p_{n}-\alpha\right)}$ and choose integers $N_{1}, N_{2}$ such that

$$
2 r<\rho \theta^{N_{1}} \leq \frac{2 r}{\theta}, \quad \frac{H_{0}}{2}<2^{N_{2}} h \leq H_{0} .
$$

Thus the inequality Eq. (2.25) can be rewritten in the form

$$
I(\rho, h) \leq A I(\theta \rho, h)+B I(\rho, 2 h)+\gamma G_{1}(r, \rho, h) .
$$

From this we deduce

$$
\begin{aligned}
I(\rho, h) \leq(2 A)^{N_{1}} \sum_{j=0}^{N_{2}-1}(2 B)^{j} I\left(2 r, 2^{j} h\right) & +(2 B)^{N_{2}} \sum_{l=0}^{N_{1}-1}(2 A)^{l} I\left(\theta^{l} \rho, H_{0}\right) \\
& +\sum_{l=0}^{N_{1}-1} \sum_{j=0}^{N_{2}-1} A^{l} B^{j} G_{1}\left(r, \theta^{l} \rho, 2^{j} h\right) .
\end{aligned}
$$

Let us estimate the terms in the right-hand side of Eq. (2.36). By Eq. (1.17) we have

$$
I\left(2 r, 2^{j} h\right) \leq \gamma\left(2^{j} h\right)^{a_{s}} M^{\alpha-1+\lambda}(r) r^{n-s} \leq \gamma\left(2^{j} h\right)^{a_{s}} r^{\alpha-\frac{\lambda(n-s-\alpha)}{\alpha-1}}\left(r^{\frac{n-s-\alpha}{\alpha-1}} M(r)\right)^{\alpha-1+\lambda},
$$


choose $c_{6}<1$ such that

$$
2^{a_{s}+1} B=2^{a_{s}+1} c_{5} c_{6}^{\alpha-\frac{n-s-\alpha}{\alpha-1}\left(p_{n}-\alpha\right)} \leq \frac{1}{2},
$$

hence Eq. (2.37) yields

$$
(2 A)^{N_{1}} \sum_{j=0}^{N_{2}-1}(2 B)^{j} I\left(2 r, 2^{j} h\right) \leq \gamma(2 A)^{N_{1}} h^{a_{s}} r^{\alpha-\frac{\lambda(n-s-\alpha)}{\alpha-1}}\left(r^{\frac{n-s-\alpha}{\alpha-1}} M(r)\right)^{\alpha-1+\lambda} .
$$

By Eq. (2.35) we have

$$
(2 A)^{N_{1}} \leq \gamma\left(\frac{\rho}{r}\right)^{(n-s) \frac{q-1}{q}+\frac{\alpha+\lambda}{\log _{2} \frac{1}{\theta}},}
$$

choosing $\theta \in(0,1)$ from the condition

$$
\frac{\alpha+\lambda}{\log _{2} \frac{1}{\theta}} \leq \alpha-(n-s) \frac{q-1}{q}-\frac{\lambda(n-s-\alpha)}{\alpha-1},
$$

we conclude from Eq. (2.38) that

$$
(2 A)^{N_{1}} \sum_{j=0}^{N_{2}-1}(2 B)^{j} I\left(2 r, 2^{j} h\right) \leq \gamma h^{a_{s}} \rho^{\alpha-\frac{\lambda(n-s-\alpha)}{\alpha-1}}\left(r^{\frac{n-s-\alpha}{\alpha-1}} M(r)\right)^{\alpha-1+\lambda} .
$$

Using Eq. (1.17) we have

$$
\begin{aligned}
I\left(\theta^{l} \rho, H_{0}\right) \leq & \gamma\left(\theta^{l} \rho\right)^{(n-s) \frac{q-1}{q}} \int_{D_{2}\left(H_{0}\right)} d x^{\prime \prime}\left(\int_{\rho\left(x^{\prime}\right) \leq \theta^{l} \rho} \rho^{-\frac{n-s-\alpha}{\alpha-1}(\alpha-1+\lambda) q}\left(x^{\prime}\right) d x^{\prime}\right)^{\frac{1}{q}} \\
& \leq \gamma\left(\theta^{l} \rho\right)^{\alpha-\frac{\lambda(n-s-\alpha)}{\alpha-1}} .
\end{aligned}
$$

The last inequality ensures that

$$
\begin{aligned}
(2 B)^{N_{2}} \quad \sum_{l=0}^{N_{1}-1}(2 A)^{l} I\left(\theta^{l} \rho, H_{0}\right) \leq \gamma(2 B)^{N_{2}} \rho^{\alpha-\frac{\lambda(n-s-\alpha)}{\alpha-1}} \\
\times \sum_{l=0}^{N_{1}-1} 2^{(\alpha+\lambda) l} \theta^{l\left(\alpha-(n-s) \frac{q-1}{q}-\frac{\lambda(n-s-\alpha)}{\alpha-1}\right)} .
\end{aligned}
$$

Choosing $\theta, c_{6}$ small enough so that

$$
\begin{gathered}
\frac{\alpha+\lambda+1}{\log _{2} \frac{1}{\theta}} \leq \alpha-(n-s) \frac{q-1}{q}-\frac{\lambda(n-s-\alpha)}{\alpha-1}, \\
2 B=2 c_{5} c_{6}^{\alpha-\frac{n-s-\alpha}{\alpha-1}\left(p_{n}-\alpha\right)} \leq 2^{-n-a_{s}+s},
\end{gathered}
$$

we conclude from Eq. (2.41) that

$$
(2 B)^{N_{2}} \sum_{l=0}^{N_{1}-1}(2 A)^{l} I\left(\theta^{l} \rho, H_{0}\right) \leq \gamma h^{n+a_{s}-s} \rho^{\alpha-\frac{\lambda(n-s-\alpha)}{\alpha-1}} .
$$


Finally from the condition Eq. (1.17) we have

$$
\begin{gathered}
A^{l} B^{j} G_{1}\left(r, \theta^{l} h, 2^{j} h\right) \leq \gamma A^{l} B^{j}\left(2^{j} h\right)^{a_{s}}\left(\theta^{l} \rho\right)^{\alpha}\left\{\left(\theta^{l} \rho\right)^{n-s-\frac{\lambda(n-s-\alpha)}{\alpha-1}}\right. \\
+\left(\theta^{l} \rho\right)^{-\frac{\lambda(n-s-\alpha)}{\alpha-1}}\left(\sum_{i=1}^{n-s}\left(r^{\frac{n-s-\alpha}{\alpha-1}} M(r)\right)^{p_{i}-1}+\sum_{i=1}^{n-s}\left(r^{\frac{n-s-\alpha}{\alpha-1}} M(r)\right)^{1-\frac{1}{p_{i}}}\right) \\
\left.+\sum_{i=n-s+1}^{n}\left(2^{j} h\right)^{-\frac{\alpha(n-s-1)}{\alpha-1}+\frac{n-s-\alpha}{\alpha-1} p_{i}}\left(r^{\frac{n-s-\alpha}{\alpha-1}} M(r)\right)^{p_{i}-1+\lambda}+\left(r^{\frac{n-s-\alpha}{\alpha-1}} M(r)\right)^{\alpha-1+\lambda}\right\} .
\end{gathered}
$$

First, choose $c_{6}$ from the condition

$$
2^{a_{s}} B=2^{a_{s}} c_{5} c_{6}^{\alpha-\frac{n-s-\alpha}{\alpha-1}\left(p_{n}-1\right)} \leq 2^{\alpha \frac{n-s-1}{\alpha-1}-\frac{n-s-\alpha}{\alpha-1}} p_{n}-1,
$$

next choose $\theta$ from the condition

$$
2^{\alpha+\lambda} \theta^{\alpha-(n-s) \frac{q-1}{q}}\left\{\theta^{n-s-\frac{\lambda(n-s-\alpha)}{\alpha-1}}+\theta^{-\frac{\lambda(n-s-\alpha)}{\alpha-1}}+1\right\} \leq \frac{1}{2},
$$

we conclude from Eq. (2.45) that

$$
\sum_{l=0}^{N_{1}-1} \sum_{j=0}^{N_{2}-1} A^{l} B^{j} G_{1}\left(r, \theta^{l} h, 2^{j} h\right) \leq \gamma G_{2}(r, \rho, h)+\gamma h^{a_{s}} \rho^{n-s+\alpha-\frac{\lambda(n-s-\alpha)}{\alpha-1}} .
$$

Combining estimates Eq. (2.36)-(2.48) we arrive at the required Eq. (2.34).

\subsection{Pointwise estimates of solutions}

Fix $\rho>0$, for $j=1,2, \ldots, J=\left[\frac{\ln \frac{R_{0}}{\rho}}{\ln \frac{1}{\theta}}\right]+1$ set $\rho_{j}=R_{0} \theta^{j}$. Let $x_{0}$ be an arbitrary point in $D\left(R_{0}, H_{0}\right) \backslash D\left(\rho_{j}, H_{0}\right)$. For $l=0,1,2, \ldots$ set $R_{l}=(1-\theta) \rho_{j}\left(1-\frac{1}{2}+\frac{1}{2^{l+1}}\right), \bar{R}_{l}=\frac{1}{2}\left(R_{l}+\right.$ $\left.R_{l+1}\right), B_{R_{l}}\left(x_{0}\right)=\left\{x: \rho\left(x^{\prime}-x_{0}^{\prime}\right) \leq R_{l}, \rho\left(x^{\prime \prime}-x_{0}^{\prime \prime}\right) \leq c_{6}^{-1} R_{l}\right\}, k_{l}=2 k_{0}-\frac{k_{0}}{2^{l}}, A_{k, R}=$ $\left\{x \in B_{R}\left(x_{0}\right): u_{\rho_{j-1}} \geq k\right\}$, here $\theta, c_{6}$ were defined in Theorem $2.2, k_{0}$ is a positive number depending on the known parameters only, which will be specified later.

Let $\xi_{l} \in C_{0}^{\infty}\left(B_{\bar{R}_{l}}\right)$ be such that $\xi_{l} \equiv 1$ for $x \in B_{R_{l+1}}\left(x_{0}\right),\left|\frac{\partial \xi_{l}}{\partial x_{i}}\right| \leq$ $\gamma 2^{l} \rho_{j}^{-\frac{\alpha}{p_{i}}-\frac{n-s-\alpha}{\alpha-1}\left(\frac{\alpha}{p_{i}}-1\right)}, i=1, \ldots, n$.

Testing Eq. (1.15) by $\varphi=\left(u_{\rho_{j-1}}-k_{l+1}\right)_{+}^{\lambda} \xi_{l}^{m-1}, \quad \psi=\xi_{l}$, using Eq. (1.2) and the Young inequality we have

$$
\begin{aligned}
& \sum_{i=1}^{n} \int_{A_{k_{l+1}, \bar{R}_{l}}}\left(u_{\rho_{j-1}}-k_{l+1}\right)^{\lambda-1}\left|u_{x_{i}}\right|^{p_{i}} \xi_{l}^{m} d x \leq \gamma 2^{\gamma l} \sum_{i=1}^{n} \rho^{-\alpha-\frac{n-s-\alpha}{\alpha-1}\left(\alpha-p_{i}\right)} \\
& \quad \times \int_{A_{k_{l+1}, \bar{R}_{l}}}\left(u_{\rho_{j-1}}-k_{l+1}\right)^{p_{i}-1+\lambda} d x \\
& +\gamma \int_{A_{k_{l+1}, \bar{R}_{l}}}\left(u_{\rho_{j-1}}-k_{l+1}\right)^{\alpha-1+\lambda} d x+\gamma\left|A_{k_{l+1}, \bar{R}_{l}}\right| .
\end{aligned}
$$


Using Eq. (1.17) we conclude from Eq. (2.49) that

$$
\sum_{i=1}^{n} \int_{A_{k_{l+1}, \bar{R}_{l}}}\left(u_{\rho_{j-1}}-k_{l+1}\right)^{\lambda-1}\left|u_{x_{i}}\right|^{p_{i}} \xi_{l}^{m} d x \leq \gamma 2^{\gamma l} \rho_{j}^{s-n-\frac{\lambda(n-s-\alpha)}{\alpha-1}}\left|A_{k_{l+1}, \bar{R}_{l}}\right| .
$$

Using Eq. (1.5), the Hölder inequality and Lemma 2.2 with $\alpha_{1}=\cdots=\alpha_{n}=1-\lambda$, we obtain

$$
\begin{aligned}
& \int_{A_{k_{l+1}, R_{l+1}}}\left(u_{\rho_{j-1}}-k_{l+1}\right)^{\alpha-1+\lambda} d x \leq \int_{A_{k_{l+1}, \bar{R}_{l}}}\left(u_{\rho_{j-1}}-k_{l+1}\right)^{\alpha-1+\lambda} \xi_{l}^{m(\alpha-1+\lambda)} d x \\
& \leq \gamma\left(\int_{A_{k_{l+1}, \bar{R}_{l}}}\left(\left(u_{\rho_{j-1}}-k_{l+1}\right) \xi_{l}^{m}\right)^{\frac{(p-1+\lambda) n}{n-p}} d x\right)^{\frac{(\alpha-1+\lambda)(n-p)}{(p-1+\lambda) n}}\left|A_{k_{l+1}, \bar{K}_{l}}\right|^{1-\frac{(\alpha-1+\lambda)(n-p)}{(p-1+\lambda) n}} \\
& \leq \gamma\left(\sum_{i=1}^{n} \int_{A_{k_{l+1}, \bar{R}_{l}}}\left(u_{\rho_{j-1}}-k_{l+1}\right)^{\lambda-1} \xi_{l}^{m(\lambda-1)}\left|\frac{\partial}{\partial x_{i}}\left(\left(u_{\rho_{j-1}}-k_{l+1}\right) \xi_{l}^{m}\right)\right|^{p_{i}} d x\right)^{\frac{\alpha-1+\lambda}{p-1+\lambda}}\left|A_{k_{l+1}, \bar{R}_{l}}\right|^{1-\frac{(\alpha-1+\lambda)(n-p)}{(p-1+\lambda) n}} \\
& \leq \gamma 2^{\gamma l} \rho_{j}^{\left(s-n-\frac{\lambda(n-s-\alpha)}{\alpha-1}\right) \frac{\alpha-1+\lambda}{p-1+\lambda}}\left|A_{k_{l+1}, \bar{R}_{l}}\right|^{1+\frac{\alpha-1+\lambda}{p-1+\lambda} \frac{p}{n}} .
\end{aligned}
$$

Using the evident inequality

$$
\left|A_{k_{l+1}, \bar{R}_{l}}\right| \leq 2^{l(\alpha-1+\lambda)} k_{0}^{-(\alpha-1+\lambda)} \int_{A_{k_{l}, R_{l}}}\left(u_{\rho_{j-1}}-k_{l}\right)^{\alpha-1+\lambda} d x
$$

and setting $y_{l}=\int_{A_{k_{l}, R_{l}}}\left(u_{\rho_{j-1}}-k_{l}\right)^{\alpha-1+\lambda} d x$ we obtain

$$
y_{l+1} \leq \gamma 2^{\gamma l} \rho_{j}^{\left(s-n-\frac{\lambda(n-s-\alpha)}{\alpha-1}\right) \frac{\alpha-1+\lambda}{p-1+\lambda}} k_{0}^{-(\alpha-1+\lambda)\left(1+\frac{\alpha-1+\lambda}{p-1+\lambda} \frac{p}{n}\right)} y_{l}^{1+\frac{\alpha-1+\lambda}{p-1+\lambda} \frac{p}{n}}, \quad l=0,1,2, \ldots
$$

Due to Lemma 2.3 this inequality implies that $y_{l} \rightarrow 0$ as $l \rightarrow \infty$ if $k_{0}$ satisfies the following condition

$$
y_{0}=\gamma \rho^{-\left(s-n-\frac{\lambda(n-s-\alpha)}{\alpha-1}\right) \frac{n}{p}} k_{0}^{(\alpha-1+\lambda)\left(\frac{n}{p} \frac{p-1+\lambda}{\alpha-1+\lambda}+1\right)} .
$$

From this we obtain that

$$
\left(\operatorname{ess} \sup \left\{u_{\rho_{j-1}}(x): x \in B_{\frac{1-\theta}{2} \rho_{j}}\left(x_{0}\right)\right\}\right)^{\alpha-1+\lambda+\frac{n}{p}(p-1+\lambda)} \leq \gamma \rho_{j}^{\left(s-n-\frac{\lambda(n-s-\alpha)}{\alpha-1}\right) \frac{n}{p}} \int_{E\left(\rho_{j-1}\right)} u_{\rho_{j-1}}^{\alpha-1+\lambda} d x .
$$

Since $x_{0}$ is an arbitrary point in $D\left(R_{0}, H_{0}\right) \backslash D\left(\rho_{j}, H_{0}\right)$, from Eq. (2.52) it follows

$$
\left(M\left(\rho_{j}\right)-M\left(\rho_{j-1}\right)\right)^{\alpha-1+\lambda+\frac{n}{p}(p-1+\lambda)} \leq \gamma \rho_{j}^{\left(s-n-\frac{\lambda(n-s-\alpha)}{\alpha-1}\right) \frac{n}{p}} \int_{E\left(\rho_{j-1}\right)} u_{\rho_{j-1}}^{\alpha-1+\lambda} d x .
$$

Using Theorem 2.2 with $\rho=\rho_{j-1}, h=c_{6}^{-1} \rho_{j-1}$ and the Hölder inequality we obtain $\left(M\left(\rho_{j}\right)-M\left(\rho_{j-1}\right)\right)^{\alpha-1+\lambda+\frac{n}{p}(p-1+\lambda)} \leq \gamma \rho_{j}^{\left(s-n-\frac{\lambda(n-s-\alpha)}{\alpha-1}\right) \frac{n}{p}}\left\{\rho_{j}^{a_{s}+n-s+\alpha-\frac{\lambda(n-s-\alpha)}{\alpha-1}}+G_{2}\left(r, \rho_{j-1}, c_{6}^{-1} \rho_{j-1}\right)\right\}$. 
Passing in Eq. (2.53) to the limit $r \rightarrow 0$, by Eq. (1.17) we obtain

$$
\left(M\left(\rho_{j}\right)-M\left(\rho_{j-1}\right)\right)^{\alpha-1+\lambda+\frac{n}{p}(p-1+\lambda)} \leq \gamma \rho_{j}^{n+\alpha-\frac{\lambda(n-s-\alpha)}{\alpha-1}+\left(s-n-\frac{\lambda(n-s-\alpha)}{\alpha-1}\right) \frac{n}{p}} .
$$

In order to complete the proof of Theorem 1.2 we sum up Eq. (2.54) with respect to $j$ from 1 to $J$

$$
M(\rho) \leq M\left(\rho_{J}\right) \leq M\left(R_{0}\right)+\gamma \rho_{J}^{-\frac{n-s-\alpha}{\alpha-1}+c} \leq M\left(R_{0}\right)+\gamma \rho^{-\frac{n-s-\alpha}{\alpha-1}+c}
$$

where $c=\frac{\frac{\alpha}{\beta}(n-s-1)-n+s+\alpha+(n-s-1)\left(n-1-\frac{n-p}{p} \alpha\right)}{(\alpha-1)\left(\alpha+1+\lambda+\frac{n}{p}(p-1+\lambda)\right)}>0$ by Eq. (1.5).

From this the required Eq. (1.18) follows, which proves Theorem 1.2.

\section{Proof of Theorem 1.1}

\subsection{Boundedness of the solutions}

For $j=0,1,2, \ldots$ set $\rho_{j}=R_{0}\left(1-\frac{1}{2}+\frac{1}{2^{j+1}}\right), \bar{\rho}_{j}=\frac{1}{2}\left(\rho_{j}+\rho_{j+1}\right), h_{j}=H_{0}(1-$ $\left.\frac{1}{4}+\frac{1}{2^{j+2}}\right), \bar{h}_{j}=\frac{1}{2}\left(h_{j}+h_{j+1}\right), k_{j}=2 k_{0}-\frac{k_{0}}{2^{j}}, A_{k_{j}, \rho_{j}, h_{j}}=\left\{x \in D\left(\rho_{j}, h_{j}\right): u \geq k_{j}\right\}$, where $k_{0}$ is a positive number depending on the known parameters only, which will be specified later. Let $\varphi_{j}\left(x^{\prime}\right) \in C_{0}^{\infty}\left(D_{1}\left(\bar{\rho}_{j}\right)\right)$ be such that $\varphi_{j}\left(x^{\prime}\right) \equiv 1$ for $x^{\prime} \in D_{1}\left(\rho_{j+1}\right)$, $\left|\frac{\partial \varphi_{j}\left(x^{\prime}\right)}{\partial x_{i}}\right| \leq \gamma 2^{j}, i=1, \ldots, n-s ; \zeta_{j}\left(x^{\prime \prime}\right) \in C_{0}^{\infty}\left(D_{2}\left(\bar{h}_{j}\right)\right)$ be such that $\zeta_{j}\left(x^{\prime \prime}\right) \equiv 1$ for $x^{\prime \prime} \in D_{2}\left(h_{j+1}\right),\left|\frac{\partial \zeta_{j}\left(x^{\prime \prime}\right)}{\partial x_{i}}\right| \leq \gamma 2^{j}, i=n-s+1, \ldots, n$. Set $\xi_{j}(x)=\varphi_{j}\left(x^{\prime}\right) \zeta_{j}\left(x^{\prime \prime}\right)$. Test (1.15) by $\varphi\left(u-k_{j+1}\right)_{+}^{\varepsilon} \xi_{j}^{m} \psi_{r}^{m-1}, \quad \psi=\psi_{r}$, where $\varepsilon$ depending on the known parameters only is small enough to be determined later. Using Eq. (1.2) and the Young inequality we have

$$
\begin{aligned}
& \sum_{i=1}^{n} \int_{A_{k_{j_{1}}, \bar{\rho}_{j}, \bar{h}_{j}}}\left(u-k_{j+1}\right)^{\varepsilon-1}\left|u_{x_{i}}\right|^{p_{i}} \xi_{j}^{m} \psi_{r}^{m} d x \leq \gamma \sum_{i=A_{k_{j+1}, \bar{\rho}_{j}, \bar{h}_{j}}^{n-s}}^{n-s}\left(u-k_{j+1}\right)^{p_{i}-1+\varepsilon}\left|\frac{\partial \psi_{r}}{\partial x_{i}}\right|^{p_{i}} \xi_{j}^{m} d x \\
& +\gamma 2^{j \gamma} \sum_{i=h_{k_{j+1}, \bar{\rho}_{j}, \bar{h}_{j}}^{n}}\left(u-k_{j+1}\right)^{p_{i}-1+\varepsilon} \psi_{r}^{m} \xi_{j}^{m-\tilde{p}} d x+\gamma \int_{A_{k_{j+1}, \bar{\rho}_{j}, \bar{h}_{j}}}\left(u-k_{j+1}\right)^{\alpha-1+\varepsilon} \psi_{r}^{m} \xi_{j}^{m} d x+\gamma\left|A_{k_{j+1}, \bar{\rho}_{j}, \bar{h}_{j}}\right| \text {. }
\end{aligned}
$$

Let us estimate the first term in the right-hand side of Eq. (3.1). By Theorem 1.2 we obtain

$$
\begin{aligned}
& \sum_{i=1}^{n-s} \int_{A_{k_{j+1}, \bar{j}_{j}, \bar{h}_{j}}}\left(u-k_{j+1}\right)^{p_{i}-1+\varepsilon}\left|\frac{\partial \psi_{r}}{\partial x_{i}}\right|^{p_{i}} \xi_{j}^{m} d x \\
\leq & \gamma \sum_{i=1}^{n-s} r^{n-s-\alpha-\frac{n-s-\alpha}{\alpha-1}\left(\alpha-p_{i}\right)} M^{p_{i}-1+\varepsilon}(r) \leq \gamma \sum_{i=1}^{n-s} r^{c\left(p_{i}-1+\varepsilon\right)-\varepsilon \frac{n-s-\alpha}{\alpha-1}} \leq \gamma r^{c\left(p_{1}-1\right)-\varepsilon \frac{n-s-\alpha}{\alpha-1}},
\end{aligned}
$$

where $c>0$ was defined in Eq. (2.55). Choosing $\varepsilon>0$ small enough so that

$$
\varepsilon=\frac{1}{2} \min \left(1, \frac{c\left(p_{1}-1\right)(\alpha-1)}{n-s-\alpha}\right)
$$


and passing to the limit $r \rightarrow 0$, by Eq. (3.1), Eq. (3.2) we obtain

$$
\begin{aligned}
& \sum_{i=1}^{n} \int_{A_{k_{j+1}, \bar{\rho}_{j}, \bar{h}_{j}}}\left(u-k_{j+1}\right)^{\varepsilon-1}\left|u_{x_{i}}\right|^{p_{i}} \xi_{j}^{m} d x \leq \gamma 2^{j \gamma} \sum_{i=1}^{n} \int_{A_{k_{j+1}, \bar{\rho}_{j}, \bar{h}_{j}}}\left(u-k_{j+1}\right)^{p_{i}-1+\varepsilon} \xi_{j}^{m-\tilde{p}} d x \\
+ & \gamma \int_{A_{k_{j+1}, \bar{\rho}_{j}, \bar{h}_{j}}}\left(u-k_{j+1}\right)^{\alpha-1+\varepsilon} \xi_{j}^{m} d x+\gamma \mid A_{k_{j+1}, \bar{\rho}_{j}, \bar{h}_{j} \mid .}
\end{aligned}
$$

Choose $q>1$ such that

$$
\frac{\tilde{p}-1+\varepsilon}{\alpha-1+\varepsilon}<q<\min \left\{\frac{(p-1+\varepsilon) n}{(\alpha-1+\varepsilon)(n-p)}, \frac{n-s}{n-s-\alpha}\right\},
$$

using the Young inequality from Eq. (3.4), Eq. (3.5) we obtain

$$
\sum_{i=1}^{n} \int_{A_{k_{j+1}, \bar{\rho}_{j}, \bar{h}_{j}}}\left(u-k_{j+1}\right)^{\varepsilon-1}\left|u_{x_{i}}\right|^{p_{i}} \xi_{j}^{m} d x \leq \gamma 2^{j \gamma}\left(\int_{A_{k_{j+1}, \rho_{j}, h_{j}}}\left(u-k_{j}\right)^{(\alpha-1+\varepsilon) q} d x+\left|A_{k_{j+1} \bar{\rho}_{j}, \bar{h}_{j}}\right|\right) .
$$

Similarly to Eq. (2.51) we have

$$
\begin{gathered}
\int_{A_{k_{j+1}, \rho_{j+1}, h_{j+1}}\left(u-k_{j+1}\right)^{(\alpha-1+\varepsilon) q} d x} \\
\leq \gamma 2^{j \gamma}\left(\int_{A_{k_{j}, \rho_{j}, h_{j}}}\left(u-k_{j}\right)^{(\alpha-1+\varepsilon) q} d x+\left|A_{k_{j+1} \bar{\rho}_{j}, \bar{h}_{j}}\right|\right)^{\frac{(\alpha-1+\varepsilon) q}{p-1+\varepsilon}}\left|A_{k_{j+1} \bar{\rho}_{j}, \bar{h}_{j}}\right|^{1-\frac{(\alpha-1+\varepsilon) q(n-p)}{(p-1+\varepsilon) n}} .
\end{gathered}
$$

Using the evident inequality

$$
\left|A_{k_{j+1} \bar{\rho}_{j}, \bar{h}_{j}}\right| \leq 2^{j(\alpha-1+\varepsilon) q} k_{0}^{-(\alpha-1+\varepsilon)} \int_{A_{k_{j} \rho_{j}, h_{j}}}\left(u-k_{j}\right)^{(\alpha-1+\varepsilon) q} d x
$$

and setting $y_{j}=\int_{A_{k_{j}, \rho_{j}, h_{j}}}\left(u-k_{j}\right)^{(\alpha-1+\varepsilon) q} d x$ we obtain

$$
y_{j+1} \leq \gamma 2^{j \gamma}\left(k_{0}^{1-\frac{(\alpha-1+\varepsilon) q(n-p)}{(p-1+\varepsilon) n}}+k_{0}^{1+\frac{(\alpha-1+\varepsilon) p q}{(p-1+\varepsilon) n}}\right)^{-(\alpha-1+\varepsilon) q} y_{j}^{1+\frac{(\alpha-1+\varepsilon) p q}{(p-1+\varepsilon) n}}, \quad j=0,1,2, \ldots
$$

Due to Lemma 2.3 this inequality implies that $y_{j} \rightarrow 0$ as $j \rightarrow \infty$ if $k_{0}$ satisfies the following condition

$$
y_{0}=\gamma k_{0}^{(p-1+\varepsilon) \frac{n}{p}}\left(k_{0}^{(\alpha-1+\varepsilon) q}+k_{0}^{-(\alpha-1+\varepsilon) q \frac{n-p}{n}}\right) .
$$

From Eq. (3.9) we get

$$
\operatorname{ess} \sup \left\{|u(x)|: x \in D\left(\frac{R_{0}}{2}, \frac{3 H_{0}}{4}\right)\right\} \leq \gamma+\gamma\left(\int_{D\left(R_{0}, H_{0}\right)}|u|^{(\alpha-1+\varepsilon) q} d x\right)^{\frac{1}{(p-1+\varepsilon) \frac{n}{p}-(\alpha-1+\varepsilon) q \frac{n-p}{p}}},
$$


this completes the proof of the boundedness of $u$ in the whole of $D\left(\frac{R_{0}}{4}, \frac{3 H_{0}}{4}\right)$.

\subsection{End of the proof of Theorem 1.1}

Let $K$ be a compact subset of domain $\Omega$. Let $\eta \in C_{0}^{\infty}(\Omega)$ be such that $\eta(x) E q . \equiv 1$ for $x \in K$.

Testing Eq. (1.15) by $\varphi=u \eta^{m} \psi_{r}^{m-1}, \psi=\psi_{r}$ using Eq. (1.2) the Young inequality, the boundedness of $u$ and passing to the limit $r \rightarrow 0$ we get

$$
\sum_{i=1}^{n} \int_{K}\left|\frac{\partial u}{\partial x_{i}}\right|^{p_{i}} d x \leq \gamma .
$$

Let $\varphi \in \stackrel{\circ}{W}^{1, \bar{p}}(\Omega)$. Test Eq. (1.15) by $\varphi \psi_{r}$, using Eq. (3.11) and the boundedness of the solution we pass to the limit $r \rightarrow 0$. So we obtain the required integral identity with an arbitrary $\varphi \in \stackrel{\circ}{W}^{1, \bar{p}}(\Omega)$ and $\psi \equiv 1$. Thus Theorem 1.1 is proved.

\section{References}

1. Besov, O., Il'in, V.P., Nikol'ski, S.M.: Integral representations of functions and embedding theorems. New York-Toronto (1978)

2. Boccardo, L., Gallouet, T., Marcellini, P.: Anisotropic equations in $L^{1}$. Differ. and Integr. Equat. 9(N 11), 209-212 (1996)

3. Fusco, N., Sbordone, C.: Some remarks on the regularity of minima of anisotropic integrals. Comm. PDE 18(N 1-2), 153-167 (1993)

4. Giaquinta, M.: Growth conditons and regularity, a counterexample. Manuscripta Math. 59, 245-248 (1987)

5. Gilberg, D., Serrin, J.: On isolated singularities of solutions of second order elliptic differential equations. J. Analyse Math. 4, 309-340 (1955)

6. Kolodij, I.M.: On boundedness of generalized solution of elliptic differential equations. Moscow University Vestnik, vol. 5, pp. 44-52 (1970)

7. Ladyzenskaya, O.A., Ural'tseva, N.N.: Linear and quasilinear elliptic equations. Academic Press, New York - London (1968)

8. Lieberman, G.: Gradient estimates for anisotropic elliptic equations, Adv. Diff. Equ. 10, 767-812 (2005)

9. Marcellini, P.: Un example de solution discontinue d'un problem variationel dans le cas scalaire, Preprint N 11, Ins. Mat, "U. Dini”, Firenze (1987)

10. Marcellini, P.: Regularity and existence of solutions of elliptic equations with $p, q$ growth conditions. J. Diff. Equat. 90, 1-30 (1991)

11. Namlyeyeva, Y.V., Shishkov, A.E., Skrypnik, I.I.: Isolated singulatities of solutions of quasilinear anisotropic elliptic equations. Advanced Nonlin. Studies. 6(N 4), 617-641 (2006)

12. Nicolosi, F., Skrypnik, I.V., Skrypnik, I.I.: Precise point-wise growth conditions for removable isolated singularities. Comm. PDE 28(3-4), 677-696 (2003)

13. Serrin, J.: Isolated singularities of solutions of quasi-linear equations. Acta Math. 113, 219-240 (1965)

14. Serrin, J.: Local behaviour of solutions of quasilinear equations. Acta Math. 111, 247-302 (1964)

15. Skrypnik, I.I.: On removabililty of singularities on manifolds for solutions of non-linear elliptic equaions. Sbornik Math. 194:9, 1361-1381 (2003)

16. Skrypnik, I.V.: On pointwise estimates of certain capacity potentials, Translations of A.M.S., Providence, RI, pp. 15-22 (1989)

17. Skrypnik, I.V.: Methods for Analysis of Nonlinear Elliptic Boundary Value Problems, Translations of A.M.S., RI, vol. 139 (1994)

18. Veron, L.: Singularity of Solutions of Second Order Quasilinear Equations. Longman, Harlow (1996) 